\title{
Electron Tomography of Frozen-Hydrated Sections
}

\author{
C.-E. Hsieh", M. Marko , J. Frank ${ }^{* * *}$, and C. A. Mannella \\ *Resource for Visualization of Biological Complexity, Wadsworth Center, Albany, NY 12201-0509 \\ **Howard Hughes Medical Institute
}

Electron tomography provides a 3-D reconstruction derived from a series of projection images recorded as the specimen is tilted in the microscope.[1] Despite the challenges of low contrast and sensitivity to beam-induced specimen damage, electron tomography can be applied to frozenhydrated biological material. This avoids the necessity for chemical fixation, dehydration, and straining, and thus allows study of the specimen in a "near-native" state. A variety of specimens have been studied in aqueous suspension by electron tomography of thin films of vitreous ice prepared by rapid plunging into a cryogen. These include microorganisms and molecules [2], mitochondria [3,4], and the skeletal muscle triad junction.[5] We have succeeded in extending this 3-D imaging technique to frozen-hydrated tissue sections, which makes it possible to study a wide range of "native" sub-cellular structures in situ.

The water in biological specimens must be frozen in "vitreous" or amorphous form in order to avoid nanometer-scale damage to the specimen due to ice crystal formation.[6] For tissue, the preferred method is high-pressure freezing, which greatly extends the depth to which good freezing can be obtained.[7] The frozen tissue must be maintained below the de-vitrification temperature $\left(\sim-140^{\circ} \mathrm{C}\right)$ throughout ultramicrotomy and microscopy. While the cutting of suitable frozen-hydrated sections is challenging, the collective experience of a few dedicated labs has led to improvements in instrumentation and technique that have greatly improved the success rate.[8] During recording of the tomographic tilt series, care must be taken not to exceed a total dose of about $5000 \mathrm{e}^{-} / \mathrm{nm}^{2}$. This avoids serious distortions in the frozen sections, and prevents damage to the higher-resolution structure of the specimen. The use of automated procedures for recording the tilt series is important for limiting the cumulative electron dose.[9]

In our experiments, freshly excised rat liver tissue was frozen in a Balzers HPC-110 high-pressure freezer. Specimen holders from the freezer were opened to expose the tissue and directly clamped in the microtome chuck. A Diatome diamond trimming knife was used to trim away the metal of the specimen holder and produce a block face $150 \mu \mathrm{m}$ on a side. Sections 160-200-nm thick were cut at $-160^{\circ} \mathrm{C}$ with a Leica UCT/ EM FCS cryo-ultramicrotome using a Diatome $35^{\circ}$ "dry" cryo knife and a Diatome Static Line adjustable ionizer. After a few ribbons of sections had accumulated at the knife edge, the ionizer was switched off, and the sections were transferred to 200-mesh folding grids using an eyelash. One grid in the attached pair had a Formvar-carbon support film on which $20 \mathrm{~nm}$ colloidal gold particles were deposited, for use as alignment fiducials. Each grid was placed on a rectangular piece of indium foil, which was then folded to form an envelope and firmly pressed flat. The indium foil provided protection for the grid during handling and long-term storage under liquid nitrogen.[10]

The indium foil envelopes were opened in the workstation of the cryotransfer specimen holder (Gatan 626), and the grids were again pressed flat before inserting them into the holder. This step was found to be important for enhancing attachment of the section to the grid, which minimized 
distortion and movement of the section during recording of the tilt series. Tilt series were recorded at $-176^{\circ} \mathrm{C}$ over a $120^{\circ}$ tilt range $\left(2^{\circ}\right.$ interval) with a JEOL JEM- $4000 \mathrm{FX}$ operated at $400 \mathrm{keV}$, using a 1024x1024-pixel CCD camera and automation software.[11] The total electron dose was 4000-4500 $\mathrm{e}^{-} / \mathrm{nm}^{2}$, the defocus was $-15 \mu \mathrm{m}$, and the pixel size was $2.1 \mathrm{~nm}$ after $2 \mathrm{x}$ binning. Tomographic reconstruction was by weighted back-projection. First results indicate that tomography can provide useful 3-D images of the biological structure inside the sections (approximate resolution $8 \mathrm{~nm}$ ), separated from the surface defects (e.g., knife marks and crevasses) that dominate the projection image.[12]

References

[1] J. Frank, Electron Tomography, Plenum, New York, 1992.

[2] W. Baumeister et al., Tr. Cell Biol. 9 (1999) 81.

[3] C.A. Mannella et al., Microsc. Microanal. 5 (Suppl. 2) (1999) 416.

[4] D. Nicastro et al., J. Struct. Biol. 129 (2000) 48.

[5] C.-E. Hsieh et al., Microsc. Microanal. 7 (Suppl. 2) (2001) 94.

[6] J. Dubochet et al., Quart. Rev. Biophys. 21 (1988) 129.

[7] D. Studer et al., J. Microsc. 179 (1995) 321.

[8] H. Sitte, Scanning Microsc. Suppl. 10 (1996) 387.

[9] A.J. Koster et al., J. Struct. Biol. 120 (1997) 276.

[10] S. Shi et al., Microsc. Res. Tech. 33 (1996) 241.

[11] B.K. Rath et al., J. Struct. Biol. 120 (1997) 210.

[12] Supported by NIH/NCRR grant RR01219. We thank Dr. Shanling Shi, Prof. Jacques Dubochet and Ashraf Al-Amoudi for advice in cutting frozen-hydrated sections.
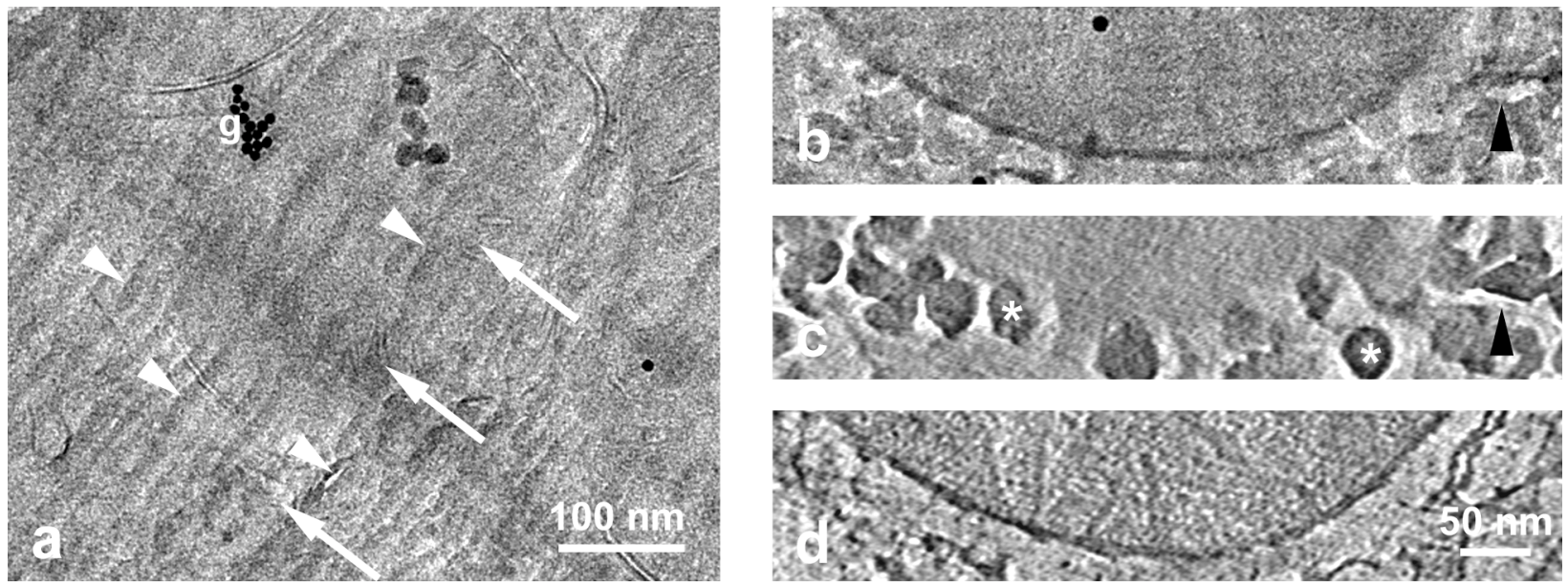

FIG. 1. a) Typical liver section with knife marks (arrows), crevasses (arrowheads), and colloidal gold (g) used for alignment. b) $0^{\circ}$ image from tomographic tilt series. c) Tomographic slice $30 \mathrm{~nm}$ from surface, crevasse (arrowhead) as in (b). d) Slice $90 \mathrm{~nm}$ from surface of 200nm-thick section. Interior of section is free of defects. 